\title{
Neep21 as an effector of the NGF-TrkA signaling endosome
}

\author{
. Kate McDaniel ${ }^{1}$, Kelly Barford ${ }^{2,3}$, Christopher Deppmann ${ }^{4}$, Bettina Winckler ${ }^{2,3}$ \\ ${ }^{1}$ UVa Undergraduate Neuroscience Major, ${ }^{2}$ UVa Neuroscience Graduate Program, ${ }^{3}$ UVa Department of Cell \\ Biology, ${ }^{4}$ VVa Department of Biology.
}

2016 Double Hoo Report

Spring 2017 


\section{TrkA and Nerve Growth Factor}

The sympathetic nervous system is made up of neurons most known for the "fight or flight" response. The axons of sympathetic neurons extend into the periphery to innervate peripheral organs. During development, peripheral organs, such as the lungs, release nerve growth factor (NGF) which is a neurotrophin essential for neuronal survival in sympathetic neurons ${ }^{1,2}$. Only a limiting amount of NGF is released by the target organ which leads to the neurotrophic factor hypothesis: neurons are overproduced during development and only those that receive proper levels of their neurotrophin will survive ${ }^{1,2}$.

TrkA, a receptor tyrosine kinase, is the high-affinity receptor for $\mathrm{NGF}^{1}$. Upon binding, NGF-TrkA signaling endosomes are endocytosed into signaling endosomes (SE) and transported retrogradley with dynein to the soma ${ }^{2-4}$. These SEs are endocytosed both through clathrin mediated mechanisms and through Pincher mediate micropinocytosis ${ }^{1,3,4}$.

NGF-TrkA signaling is required for survival and can signal both on the axonal surface and following endocytosis ${ }^{1,2,4}$. NGF does stay bound to TrkA in SEs ${ }^{3}$. Additionally, NGF-TrkA SEs not only promote survival within the neuron, but also trigger apoptosis in surrounding cells ${ }^{1,2}$. NGF-TrkA signaling is needed for dendritic arborization, as well as pre- and postsynaptic specializations ${ }^{1,5}$. Interestingly, loss of NGF leads to apoptosis during development but only to phenotypic changes in later stages ${ }^{2,6}$.

\section{Signaling}

Multiple signaling pathways are activated by NGF-TrkA such as PI3K/Akt, Ras/MAPK and the PLC $\gamma$ pathways ${ }^{1,4}$. PI3K is not necessary for internalization but is required for retrograde signaling of NGF-TrkA SEs as well as for postsynaptic density (PSD) formation ${ }^{4,5}$. MAPK signaling is triggered upon SEs reaching the soma and is also necessary for the formation of 
$\operatorname{PSDs}^{4,5}$. For transcriptional changes in the neuron, signaling is required for long periods of time with most NGF-TrkA SEs surviving for approximately 6 hours and up to $25^{7}$.

\section{Trafficking}

Three main trafficking pathways for TrkA or NGF-TrkA have been elucidated:

anterograde transcytosis from the soma to the axon (pathway 1), retrograde transport from the growth cone to the soma (pathway 2), and the signaling transcytosis pathway (pathway 3$)^{7,8}$

(Figure 1). The signaling transcytosis pathway occurs when signaling endosomes are exocytosed onto the soma upon arriving after retrograde transport and then re-endocytosed ${ }^{7,8}$.

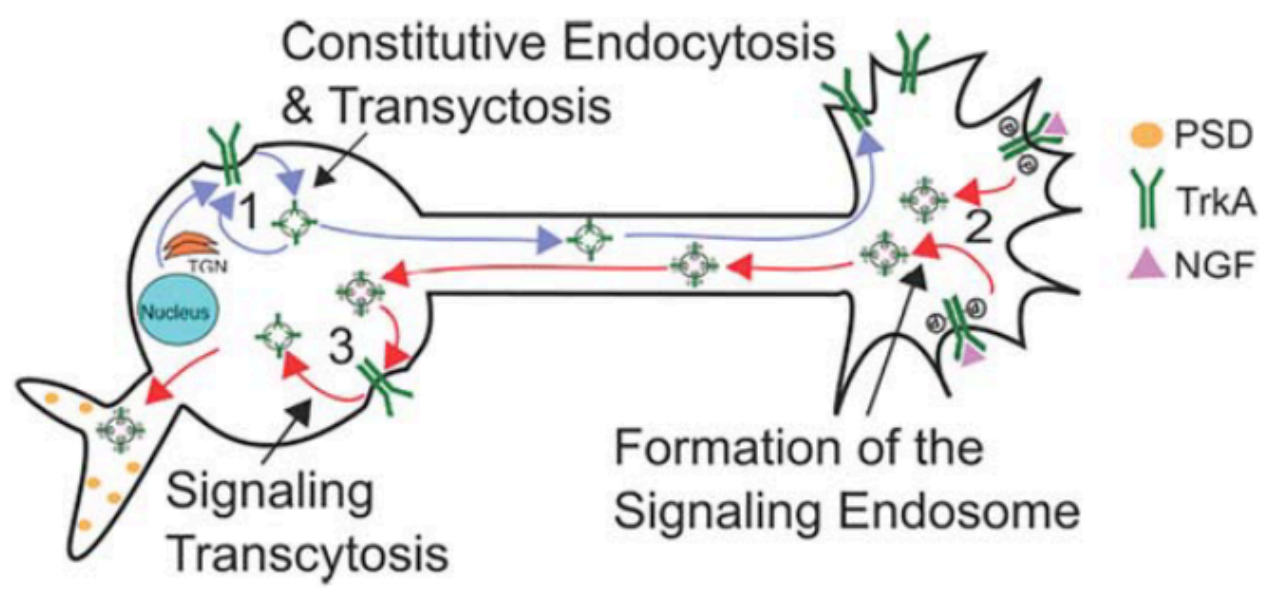

Figure 1: Three trafficking pathways for the NGF-TrkA signaling endosome. 1) Constitutive endocytosis and transcytosis is used to traffic TrkA receptors to the axon. 2) Signaling endosomes are endocytosed in the axon terminals and retrogradely trafficked back to the soma. 3) Signaling transcytosis is a process through which NGF-TrkA signaling endosomes are exocytosed onto the soma and subsequently re-endocytosed. PSDs are postsynaptic densities. From Barford et al., Devel. Neurobio 2017².

Coronin la, a structural protein that interacts with cytoskeletal proteins, is an effector of $\operatorname{TrkA}^{8}$. It is associated with TrkA and helps to prevent NGF-TrkA SE degradation as well as to promote signaling transcytosis ${ }^{4,7,8}$. Work in the Deppmann lab elucidated the signaling transcytosis pathway, as well as the involvement of Coronin $1 \mathrm{a}^{8}$. 


\section{Neep21}

Neuron enriched endosomal protein of $21 \mathrm{kD}$ (Neep21) is an endosomal protein associated with Sytaxin13, a SNARE protein localized to recycling endosomes ${ }^{9}$. Neep21 is a single pass transmembrane protein ${ }^{9}$. It is specific to the somatodendritic region of neurons and is shown to associate with Rab4, a marker of recycling endosomes, but not Rab7, a marker of late endosomes $^{9}$. When Neep21 is down-regulated, recycling of the transferrin receptor is delayed ${ }^{9}$.

Neep21 plays a role in the recycling of L1-NgCAM, a cell adhesion molecule ${ }^{10}$. Downregulation of Neep21 affects $\mathrm{L} 1-\mathrm{NgCAM}$ trafficking as without Neep21, L1-NgCAM is trafficked to the lysosome and prematurely degraded ${ }^{10}$. In the central nervous system, Neep 21 is more abundant in development than in later stages and is involved in recycling mechanisms in the somatodendritic region of neurons ${ }^{7,9,10}$. As TrkA can last for up to 25 hour in the soma and can undergo a variety of trafficking pathways, recycling and the prevention of lysosomal fusion are of great interest ${ }^{7}$.

\section{Neep21 and TrkA}

Currently, there much is still unknown about the trafficking patterns and endosomal identity of the NGF-TrkA signaling endosome. Coronin1a is the only known effector of TrkA in the soma, assisting with preventing Neep21 from undergoing lysosomal fusion ${ }^{8}$. We sought to investigate other regulators of the TrkA in the soma and chose Neep21 based on evidence the Neep 21 can regulate trafficking in the soma ${ }^{10}$. We have preliminary data showing that Neep21 and NGF-Trka co-localize in signaling endosomes (Figure 2). We aimed to investigate the role of Neep21 on NGF-TrkA signaling endosomes to 1) determine if Neep21 helps NGF-TrkA SEs avoid lysosomal fusion and 2) to determine if Neep21 is necessary for the survival of neuronal survival and development. The purpose of this project was to investigate the role of Neep21 in 
the trafficking of NGF-TrkA signaling endosomes and the survival and development of neurons.

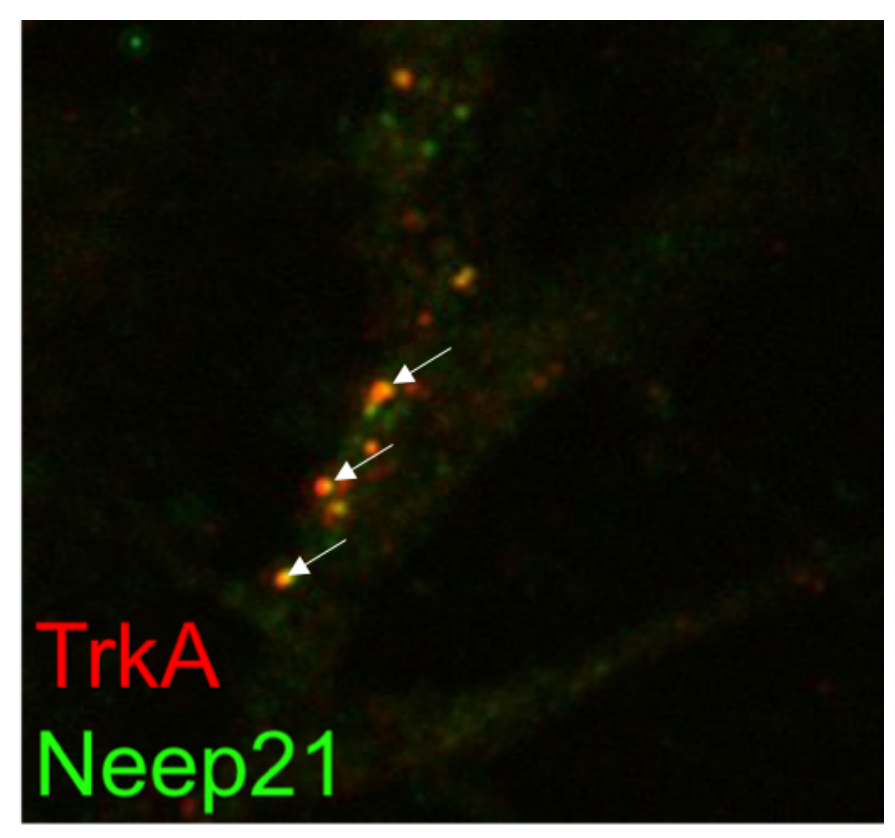

Figure 2: Neep21 and TrkA co-localize in signaling endosomes. Arrowheads show colocalization between Neep21-positive signaling endosomes and TrkA-positive signaling endosomes. 5DIV culture.

\section{Methods}

Model: We have a FLAG-TrkA knock-in mouse provided by the Deppmann lab that has a FLAG tag endogenously knocked-in on the extracellular domain of the TrkA receptor ${ }^{5}$. This allows for easy staining and visualization of the TrkA signaling endosome. Additionally, we have Neep21 wild type and Neep21 knock out mice. Neurons from the superior cervical ganglia of mice were dissected at P0 and plated in microfluidic devices. Cultures were grown for 7 days in-vitro (DIV) to allow for full growth and determination of axons and dendrites. Experiments were run at 7 DIV.

Microfluidic Devices: We used microfluidic devices that separate the cell body and distal axons in culture ${ }^{11}$. These devices allow for differential treatment of the cell body and distal axons in experiments. Microfluidic devices were made with a mix 9:1 ratio of silicon elastomer to curing 
agent poured into a 9 mold plate and baked overnight at $60^{\circ} \mathrm{C}^{8,11}$. Microfluidics are then cut out of the mold and cut into individual microfluidic devices.

Antibodies: Anti-FLAG M1 antibody (Sigma Product \#: F3040); Neep21 antibody (not commercial); MAP2 antibody (Encor Product \#P3008).

FLAG-Feeding Assay: Anti-FLAG M1 antibody (Sigma Product \#: F3040) was added to the distal axon chambers of the microfluidic chamber for 30 minutes, washed away, and chased for 2 or 6 hours. This feeding allows for visualization of NGF-TrkA signaling endosomes in the soma through immunofluorescence. To visualize SEs that undergo signaling transcytosis, a secondary stain to the anti-FLAG antibody, a donkey anti-mouse 647 antibody (called Cy5 from here out), will be used in the cell body chambers to visualize SEs that are exocytosed and re-endocytosed. Staining: Immunohistochemistry was used to visualize the soma and dendrites (MAP2). Imaris: Data analysis was completed using Imaris software. The soma was masked based off of MAP2 staining. Baseline fluorescence levels were set based on the $\mathrm{t}=0$ time-point and colocalizations dots were created based off of high-fluorescence levels in the FLAG and Neep21 channels. Dots within $0.5 \mu \mathrm{m}$ were considered to be co-localized.

\section{Results}

\section{Neep21 does not affect the number of NGF-TrkA signaling endosomes}

Coronin1a is the only known somatic effector of the TrkA receptor through regulation of lysosomal fusion ${ }^{8}$. With data showing that Neep21 is present in the somatodendritic region and affecting recycling pathways, and preliminary data demonstrating a Neep21 - TrkA colocalization, we sought to elucidate the role of Nee21 in NGF-TrkA signaling endosomes ${ }^{9,10}$. In this experiment, the FLAG-feeding assay was performed at 7DIV. Endosomes were allowed to accumulate in the soma for 2 or 6 hours. Following staining, fixation, and imaging, Imaris was 
used to determine the number of retrograde signaling endosomes per cell. The somatodendritic region was masked off of MAP2 staining. Through the masking and co-localization procedures, the total number of FLAG-positive signaling endosomes in the soma were counted. There was no significant difference in the number of NGF-TrkA signaling endosomes in Neep $21^{\mathrm{wt}}$ and Neep $21^{\mathrm{KO}}$ mice $(\mathrm{p}=0.2, \mathrm{n}=4-5$ experiments; $\mathrm{t}$-test) (Figure 3$)$.

\section{Neep21 affects the trafficking route of NGF-TrkA signaling endosomes}

As there was no change in the total number of signaling endosomes in Neep $21^{\mathrm{KO}}$ as compared to Neep $21^{\mathrm{wt}}$, we sought to investigate any difference in trafficking pattern. Using the FLAG-feeding assay with the addition of Cy5 to the cell bodies, we investigated if Neep21 has an effect on the signaling transcytosis pathway. At 6 hours, there was an increase in the number of Cy5 positive endosomes in Neep $21^{\mathrm{KO}}$ mice, suggesting that Neep 21 might play a role regulating signaling transcytosis (Figure 3). The accumulation of signaling endosomes at 6 hours in Neep $21^{\mathrm{KO}}$ neurons suggests a role for Neep21 in the trafficking of NGF. In these neurons, the increase in the number of signaling endosomes that have undergone signaling transcytosis in the cell at 6 hours suggests that in the absence of Neep21, signaling endosomes are undergoing signaling transcytosis at higher levels than in wild-type neurons. Further investigation is necessary as this phenotype was not consistent throughout all experiments. 

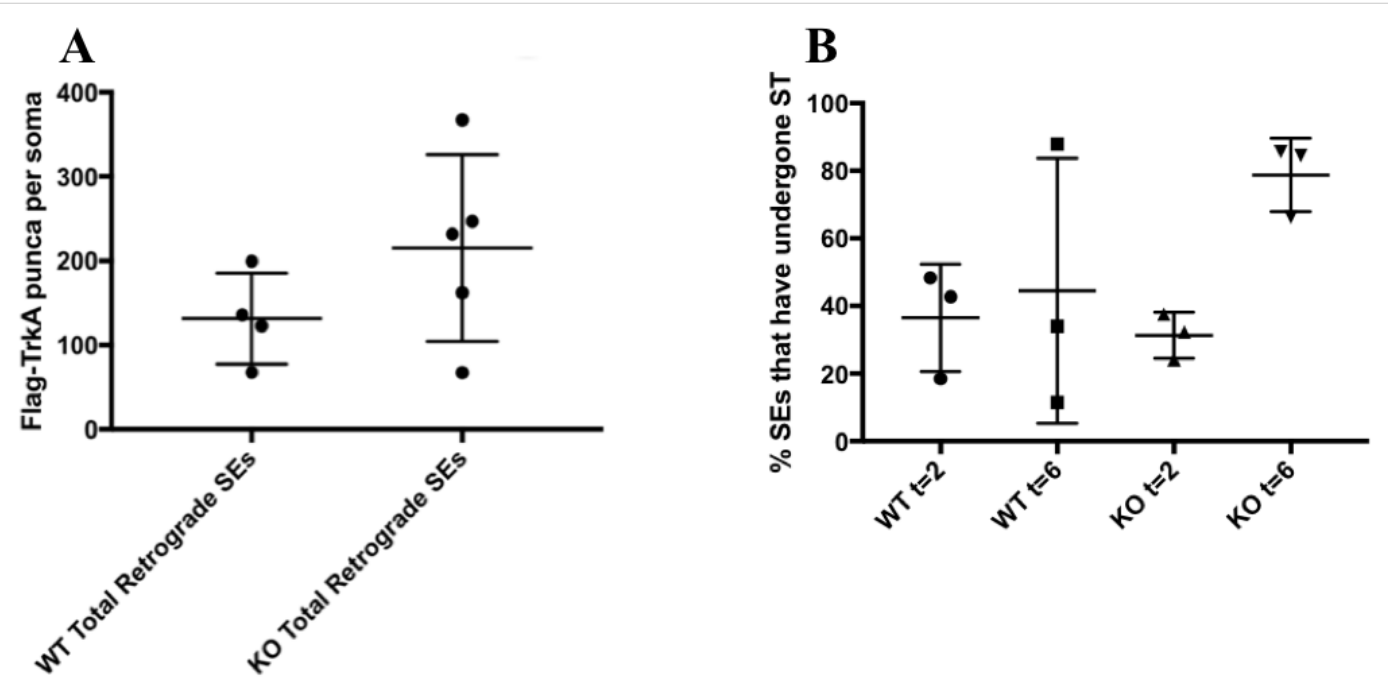

Figure 3: Neep21 ${ }^{\mathrm{KO}}$ does not affect the total number of signaling endosomes but might affect trafficking. A: There is no change in the total number of FLAG-TrkA signaling endosomes in the soma in Neep $21^{\mathrm{KO}}$ neurons compared to Neep $21^{\mathrm{wt}}$ neurons $(\mathrm{p}=0.2,4-5$ experiments; $\mathrm{t}$-test). B: In some experiments, a difference in the level endosomes that have undergone signaling transcytosis (shown by Cy5 positivity) is demonstrated after a 6 hour time course in Neep $21^{\mathrm{KO}}$ cultures ( $\mathrm{t}=$ hours; $\mathrm{n}=3$ experiments per condition).

\section{Discussion}

In these experiments, we have investigated the effect of Neep21 on the NGF-TrkA signaling endosome. We found that Neep21 is not essential for the survival of these signaling endosomes, as the loss of Neep21 did not have a significant effect on the total number of signaling endosomes. Interestingly, the loss of Neep21 increased signaling endosomes entering the signaling transcytosis pathway after a 6 hour time course, although this was not consistently seen. Neep21 has been reported to play a role in aiding recycling of L1-NgCAM and is compartmentalized to the somatodendritic domain ${ }^{10}$. Further investigation is required to fully elucidate the role of Neep21 on NGF-TrkA signaling endosome trafficking.

Investigating Neep21 in the context of NGF-TrkA signaling is difficult in that there is still much unknown about both Neep21 and NGF-TrkA signaling and trafficking. As a result, there are difficulties in isolating variables and determine what is affecting phenotypes as research 
about Neep21 is still in preliminary stages and there is no consensus on many aspects of the NGF-TrkA signaling endosome.

Further research on the NGF-TrkA signaling pathway could investigation of the Rab protein identify of NGF-TrkA signaling endosomes. The role of Rab proteins in NGF-TrkA signaling endosomes has also not been fully elucidated. While Rab5, Rab7, and Rab11 have been implicated, it is unclear which Rab proteins associate with the SE during each point of the trafficking route ${ }^{1,3,4,7,12}$. Additionally, NGF-TrkA signaling is required for the formation of postsynaptic densities in dendrites ${ }^{5}$. In addition to implications for signaling transcytosis, it is important to fully understand the identity of the NGF-TrkA signaling at all points of its trafficking pathway, how Rab identity affects those systems, and how both identity and trafficking play a role in postsynaptic density formation.

Further investigation is necessary into these pathways to fully understand the effects of the NGF-TrkA signaling endosomes on developing cells. Additionally, these experiments in conjunction with Neep $21^{\mathrm{wt}}$ and Neep $21^{\mathrm{KO}}$ mice will provide more context into the role of Neep21 in somatodendritic signaling endosomal trafficking. 
Bibliography

1. Harrington, A. W. \& Ginty, D. D. Long-distance retrograde neurotrophic factor signalling in neurons. Nat Rev Neurosci 14, 177-187 (2013).

2. Huang EJ, R. L. Neurotrophins: Roles in Neuronal Development and Function. Annu. Rev. Neurosci. 24, 677-736 (2001).

3. Delcroix, J.-D. et al. NGF Signaling in Sensory Neurons. Neuron 39, 69-84 (2003).

4. Yamashita, N. \& Kuruvilla, R. Neurotrophin signaling endosomes: Biogenesis, regulation, and functions. Curr. Opin. Neurobiol. 39, 139-145 (2016).

5. Sharma, N. et al. Long-Distance Control of Synapse Assembly by Target-Derived NGF. Neuron 67, 422-434 (2010).

6. Patapoutian, A. \& Reichardt, L. F. Trk receptors: Mediators of neurotrophin action. Curr. Opin. Neurobiol. 11, 272-280 (2001).

7. Barford, K., Deppmann, C. \& Winckler, B. The neurotrophin receptor signaling endosome: where trafficking meets signaling. Dev. Neurobiol. 1-26 (2016). doi:10.1002/dneu.22427

8. Suo, D. et al. Coronin-1 is a neurotrophin endosomal effector that is required for developmental competition for survival. Nat. Neurosci. 17, 36-45 (2014).

9. Steiner, P. et al. Modulation of receptor cycling by neuron-enriched endosomal protein of 21 kD. J. Cell Biol. 157, 1197-1209 (2002).

10. Yap, C. C. et al. The somatodendritic endosomal regulator NEEP21 facilitates axonal targeting of L1/NgCAM. J. Cell Biol. 180, 827-842 (2008).

11. Park, J. W., Vahidi, B., Taylor, A. M., Rhee, S. W. \& Jeon, N. L. Microfluidic culture platform for neuroscience research. Nat. Protoc. 1, 2128-36 (2006). 
12. Zhang, K. et al. Defective axonal transport of Rab7 GTPase results in dysregulated trophic signaling. J. Neurosci. 33, 7451-62 (2013). 